\title{
Reverse nonequilibrium molecular dynamics calculation of the Soret coefficient in liquid heptane/benzene mixtures
}

\author{
Pavel Polyakov, ${ }^{1, *}$ Florian Müller-Plathe, ${ }^{2}$ and Simone Wiegand ${ }^{1, \dagger}$ \\ ${ }^{1}$ Forschungszentrum Jülich GmbH, \\ IFF - Weiche Materie, D-52428 Jülich, Germany \\ ${ }^{2}$ Eduard-Zintl-Institut für Anorganische und Physikalische Chemie, \\ Technische Universität Darmstadt, \\ Petersenstrasse 20 D-64287 Darmstadt, Germany
}

(Dated: September 25, 2008)

\begin{abstract}
We studied the thermal diffusion behavior of mixtures of benzene and heptane isomers by reverse nonequilibrium molecular dynamics. For $n$-heptane/benzene mixtures we investigated the concentration dependence of the Soret coefficient. The Soret coefficient for equimolar mixtures of the three heptane isomers 3-methylhexane, 2,3-dimethylpentane and 2,4-dimethylpentane in benzene has been calculated. Compared to the experimental data, the simulation results show the same trend in dependence of the mole fraction and degree of branching. The negative Soret coefficient indicates the enrichment of alkanes in the cold side. In the case of the heptane isomers in benzene we could study the influence of the difference in shape and size on the thermal diffusion behavior at constant mass. In the simulation as well as in the experiment we found that the Soret coefficients becomes higher with increasing degree of branching. Such behavior can not be explained only by mass and size effects. The effect of the molecular shape needs to be considered additionaly.
\end{abstract}




\section{INTRODUCTION}

Thermal diffusion describes the migration of molecules in a temperature gradient. In the simple case of a binary mixture with constant pressure there is a mass diffusion current

$$
j_{\mathrm{D}}=-\rho D \nabla x
$$

and a thermal diffusion current $j_{\mathrm{T}}=-\rho D_{\mathrm{T}} x(1-x) \nabla T$, with $x$ the molar fraction, $\rho$ the density of the liquid, and $D$ and $D_{\mathrm{T}}$ the mutual mass and thermal diffusion coefficients, respectively. In the stationary state the two flows cancel and the resulting concentration gradient is given by

$$
\nabla x=-S_{\mathrm{T}} x(1-x) \nabla T
$$

$S_{\mathrm{T}}=D_{\mathrm{T}} / D$ is the Soret coefficient. A positive Soret coefficient of the component with the mole fraction $x$ implies that this component moves to the cold region of the fluid. The main practical applications are separation processes ${ }^{1,2}$ such as thermal field flow fractionation of polymers and colloids or isotope separation, characterization of geochemical processes ${ }^{3,4}$ and combustion $^{5}$.

The reverse nonequilibrium molecular dynamics (RNEMD) method has been developed to calculate the Soret coefficient. The energy flux is fixed by the boundary conditions and $S_{\mathrm{T}}$ can be easily calculated from temperature and concentration gradients according to Eq. 2 . This method has been successfully applied for investigation of the thermal diffusion behavior in Lennard-Jones fluids ${ }^{6}$, methane in "super" methane ${ }^{7}$, methane $/ n$-decane ${ }^{8}$, methane $/ n$ alkane $^{9}, n$-pentane/n-decane ${ }^{10}$, benzene/cyclohexane ${ }^{11}$ and water/alcohol ${ }^{12}$ mixtures. Previously we also investigated the thermal diffusion process in binary mixtures of simple molecules (tetraethylsilane, di-tert-buthylsilane and carbon tetrabromide in carbon tetrachloride) by thermal diffusion forced Rayleigh scattering (TDFRS) and MD simulations ${ }^{13}$. It was found, that the component with the larger mass and larger Hildebrandt parameter moves to the cold side. This is the typical behavior of ideal solutions of spherical molecules, for which the enthalpy of mixing and the mixing volume are close to zero. The Hildebrandt parameter provides a numerical estimate of the degree of interaction between materials, and can be a good indication of solubility. For organic compounds it can be estimated according to $\delta=\sqrt{\rho\left(H^{\text {vap }}-R T\right) / M}$ with the gas constant $\mathrm{R}$ and enthalpy of vaporization $H^{\text {vap }}$.

However, this simple rule of thumb fails for alkane/benzene mixtures ${ }^{14}$. The heavier linear 
alkane always moves to the warm side. This tendency becomes weaker with increasing degree of branching and the highly branched isomer of heptane (2,2,3 - TMB) moves to the cold side. The thermal diffusion behavior of linear alkanes is well described by a simple lattice model $(\mathrm{SLM})^{14}$. At the same time the SLM is not capable to describe the thermal diffusion behavior of branched alkanes because their thermodynamic parameters such as density, heat capacity and thermal expansion coefficient are not sensitive to the degree of branching. In this work we investigated the dependence of the transport properties versus concentration for $n$-heptane/benzene mixture and versus the degree of branching for equimolar branched heptane/benzene mixtures by RNEMD simulation. The obtained simulation results have been compared with the data from the TDFRS experiment. The TDFRS method works with fairly small temperature differences in the order of micro to milli Kelvin and is one of the most effective methods existing today for investigation of the thermal diffusion behavior of different binary mixtures. This method avoids convection and provides accurate and reliable Soret and thermal diffusion coefficients for different kinds of liquid mixtures such as low molecular weight mixtures but also polymer solutions and colloidal suspensions ${ }^{15}$.

\section{COMPUTATIONAL DETAILS}

Reverse nonequilibrium molecular dynamics method has been applied to investigate the thermal diffusion of alkane/benzene mixtures. A detailed description can be found elsewhere $^{11}$. The intermolecular force field contained constraints, angle bending, torsional potentials and harmonic dihedral potential. Lorentz-Berthelot mixing rules were employed for unlike nonbonded interactions. The force field parameters for benzene were taken from Milano et $\mathrm{al}^{16}$. The $\mathrm{C}-\mathrm{H}$ bonds were slightly polarized in order to reproduce the benzene quadrupole moment. The same benzene model has been successfully used to simulated the thermal difusion properties of benzene/cyclohexane mixtures ${ }^{11}$. For alkanes we used the TraPPE-UA ${ }^{17}$ force field. All $\mathrm{CH}_{n}$ groups were treated as individual atoms without taking into account electrostatic interactions. We also tried to use the force field from $\mathrm{Nath}^{18}$, but the obtained values for the enthalpy of vaporization were also lower than in the experiment. Chang and Sandler ${ }^{19}$ proposed a full atom force field in order to solve this problem. We are aware of the fact that the choice of the force field is crucial for calculating the thermal diffusion properties and more sophisticated models such as full atom force fields might be 
feasible in future simulations.

All systems were simulated at $T=303 \mathrm{~K}$ and $P=1$ atm. The YASP package ${ }^{20}$ was used. The cutoff length for nonbonded interactions was $1.1 \mathrm{~nm}$. The time step was $2 \mathrm{fs}$. First, the studied mixture was equilibrated in the cubic simulation box. Then, the cell was replicated in $z$-direction $\left(\mathrm{L}_{x}=\mathrm{L}_{y}=\mathrm{L}_{z} / 3 \approx 4-4.4 \mathrm{~nm}\right)$. The diffusion coefficients were obtained from equilibrium molecular dynamics simulations (EMD) with a runtime of 350 ps. The self diffusion coefficient $D^{S}$ was calculated from the mean square displacements $\triangle r^{2}$ of the center of mass of the molecules via the Einstein relation in pure liquids ${ }^{11}$

$$
D^{S}=\frac{1}{6} \frac{d}{d t}\left\langle\triangle r^{2}\right\rangle
$$

The same expression has also been used the calculated the self diffusion in binary mixtures, which is often denoted as tracer diffusion. The mutual diffusion coefficient $D_{12}$ was calculated from the mean-squared displacement of the center of mass of all molecules of one species ${ }^{11}$

$$
D_{12}^{i}=x_{1} x_{2}\left(\frac{1}{x_{1} M_{1}}+\frac{1}{x_{2} M_{2}}\right)^{2}\left(x_{i} M_{i}\right)^{2} \frac{N\left\langle\left(\triangle r_{i}^{c m}\right)^{2}\right\rangle}{6 t},
$$

where $D_{12}^{i}$ is the diffusion coefficient, calculated from the center of mass of all molecules of the species $i, x_{i}$ and $M_{i}$ the corresponding mole fraction and molecular weight, respectively and $N$ is the total number of molecules. $D_{12}^{1}$ is equal to $D_{12}^{2}$ as far as the momentum is conserved. In Eq. 1 the mutual diffusion coefficient is denoted simplified as $D$. The error bars were calculated from the standard deviation among the $x, y$ and $z$ components of the diffusion coefficient. The heat of vaporisation was calculated from the intermolecular nonbonded energy $H_{v a p}=-\left\langle E^{\text {inter }}\right\rangle+R T$.

All reverse nonequilibrium molecular-dynamics (RNEMD) simulations were performed at constant NVT conditions with 960 or 1500 molecules in the simulation box. The periodic system was divided into 20 slabs along the $z$ - direction. The average temperature $300 \mathrm{~K}$ was kept constant by a thermostat of Berendsen et al. ${ }^{21}$, with the temperature coupling time being $\tau=50$ ps. The temperature gradient was created by exchanging every $\mathrm{N}_{\text {exch }}$ steps the center of mass velocity vector of two molecules ("coldest" molecule in the hot slab one and the "hottest" molecule in the cold slab 11) of the same kind. After the concentration gradient is induced the Soret coefficient can be calculated (c.f. Eq. 2). For each simulation run two values of the Soret coefficient were calculated: from 9 slabs of the downward branch and from 9 slabs in the upward branch. The hottest and coldest slabs have been excluded 
from the analysis. The final value of $S_{\mathrm{T}}$ represents the average value, the error bars reflect the difference between $S_{\mathrm{T}}$ from downward and upward branches.

\section{EQUILIBRIUM MOLECULAR DYNAMICS SIMULATIONS}

Fig. 1A shows a good agreement between the simulated and experimental ${ }^{22}$ densities for n-heptane/benzene mixture at different concentrations. Typically, the agreement is better than $1.5 \%$. The self diffusion coefficients of benzene and $n$-heptane are $30 \%$ lower and $20 \%$ larger, respectively than the experimental value $\mathrm{e}^{16,23}$. The mutual diffusion coefficient for a $n$-heptane mole fraction of 0.25 is in satisfactory agreement with the experiment, while the other two values ( $n$-heptane mole fraction of 0.5 and 0.75 ) are systematically $\approx 40 \%$ larger than in the experiment. Nevertheless, the experimental trend is reproduced.

Table I shows the experimental and the simulation density and heat of vaporization for pure benzene, $n$-heptane, 3-methylhexane (3-MH), 2,3-dimethylpentane (2,3-DMP) and 2,4-dimethylpentane (2,4-DMP). The simulated densities are in satisfactory agreement with the experimental values. The branching effect has almost no influence on the heptane density. The vaporization enthalpies from simulations for benzene and heptane are also in satisfactory agreement with experiment, while their values become systematically smaller than in experiment with increasing degree of branching. Unfortunately, we did not find the values of the self diffusion coefficient of $n$-heptane and its isomers in the literature. The simulated self diffusion coefficient decreases with increasing degree of branching $(4.3,4.15$, 3.58 and $3.78 \mathrm{~cm}^{2} \mathrm{~s}^{-1}$ for $n$-heptane, 3-MH, 2,3-DMP and 2,4-DMP, respectively).

Fig. 2A and $\mathrm{B}$ shows the density and diffusion coefficients for equimolar mixtures of $n$ heptane and its isomers in benzene. The densities of the branched heptane/benzene mixtures are roughly $2 \%$ larger than for the equimolar heptane/benzene mixture. The calculated mutual diffusion coefficient for branched heptane/benzene mixtures agrees better with the experimental values than for the equimolar mixture of heptane in benzene. More ideal packing of the heptane isomers in benzene can be responsible for the smaller error bars of the mutual diffusion coefficient. 


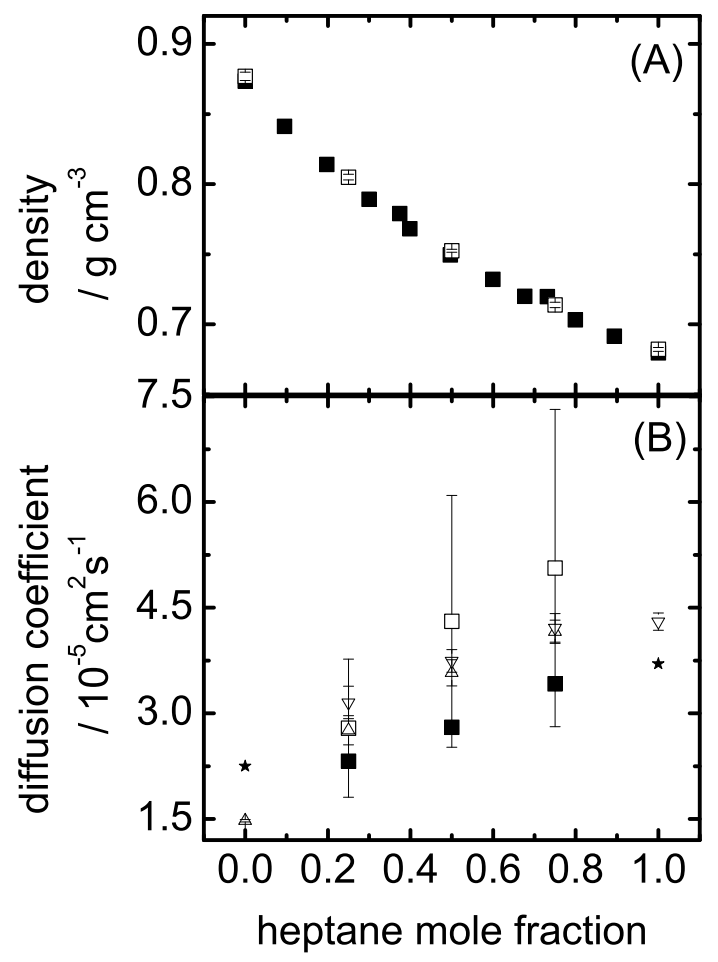

FIG. 1: (A) Comparison between simulated $(\square)$ and experimental $(\boldsymbol{\square})^{22}$ densities for $n$ heptane/benzene mixtures. (B) Comparison between simulated ( $\square$ ) and experimental ( $\square)^{14}$ mutual diffusion coefficients for $n$-heptane/benzene mixtures. Tracer and self diffusion coefficient of benzene $(\triangle)$ and $n$-heptane $(\nabla)$ in the mixture and in the pure liquid, respectively. Stars $(\star)$ represent the literature data for self diffusion coefficient $D^{S}$ of benzene ${ }^{16}$ and $n$-heptane ${ }^{23}$.

TABLE I: The density and the heat of vaporization of the investigated solvents from the experiment ${ }^{24,25}$, and simulations.

\begin{tabular}{|l|r|r|r|r|}
\hline substance & $\begin{array}{l}\rho_{\text {exp }} / \\
\mathrm{gcm}^{-3}\end{array}$ & $\begin{array}{l}\rho_{\text {sim }} / \\
\mathrm{gcm}^{-3}\end{array}$ & $\begin{array}{r}H_{\text {exp }}^{\text {vap }} / \\
\mathrm{kJ} \mathrm{mol}^{-1}\end{array}$ & $\begin{array}{r}H_{\text {sim }}^{\text {vap }} / \\
\mathrm{kJ} \mathrm{mol}^{-1}\end{array}$ \\
\hline$n$-heptane & 0.684 & 0.682 & 36.2 & 33 \\
3 -MH & 0.687 & 0.686 & 35.2 & 27.8 \\
$2,4-\mathrm{DMP}$ & 0.673 & 0.681 & 33.1 & 23.6 \\
2,3-DMP & 0.695 & 0.690 & 34.1 & 21.9 \\
benzene & 0.874 & 0.877 & 34.2 & 32.5 \\
\hline
\end{tabular}




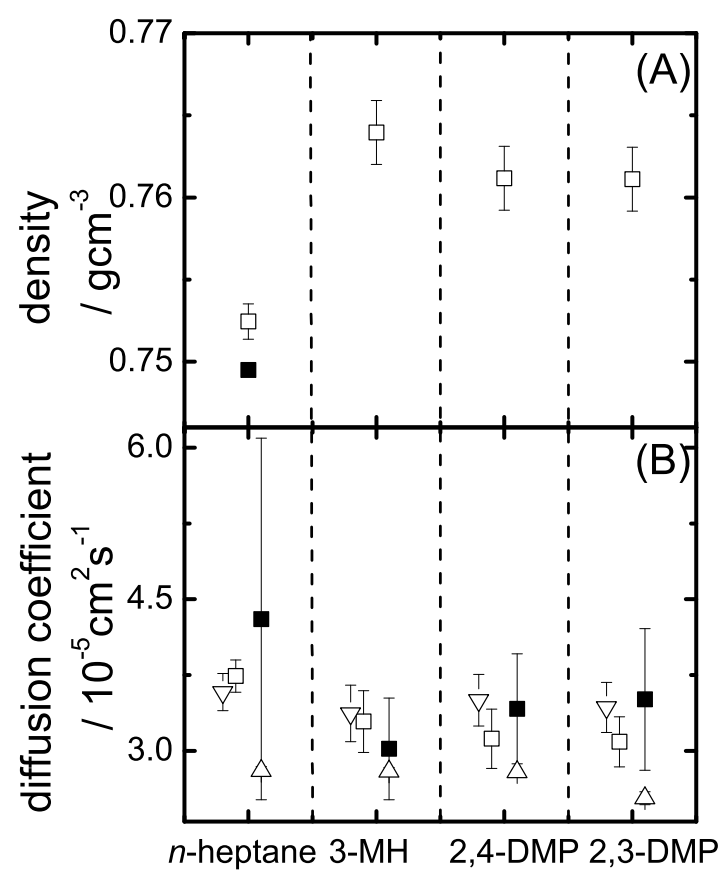

FIG. 2: (A) The simulated ( $\square$ ) and experimental (ם) alkane densities for equimolar mixtures of $n$-heptane and its isomers in benzene. (B) Comparison between simulated and experimental ${ }^{14}$ mutual diffusion coefficients $(\square, \boldsymbol{\square})$ for the same mixtures and the tracer diffusion coefficients of benzene $(\triangle)$ and of heptane and its isomers $(\nabla)$. Solid symbols refer to experimental values and open symbols to simulation results. For clarity the diffusion coefficient have been shifted by a small amount in the $\mathrm{x}$-direction.

\section{NON EQUILIBRIUM MOLECULAR DYNAMICS SIMULATIONS}

Fig. 3 shows the temperature and mole fraction profiles for three $n$-heptane/benzene mixtures with a molar fraction of $x_{\text {heptane }}=0.25,0.5$ and 0.75 . The average fluctuation of the concentration is $8 \%$ and temperature variation is $2 \mathrm{~K}$ in each slab. The simulations for $n$-heptane/benzene mixture with $x_{\text {heptane }}=0.25$ and 0.5 were performed with $N_{\text {exch }}=100$ and 1500 molecules in the simulation box. The simulation time was 11 and 13.7 ns, respectively. For $x_{\text {heptane }}=0.75$ the exchange period was 250 and the number of molecules in simulation box was equal to 960 with a simulation time was 27 ns. For this concentration it was difficult to get a linear concentration gradient at lower exchange number 220 and 125. Fig. 4 shows the calculated Soret coefficient in comparison with experimental data ${ }^{14}$. The RNEMD 


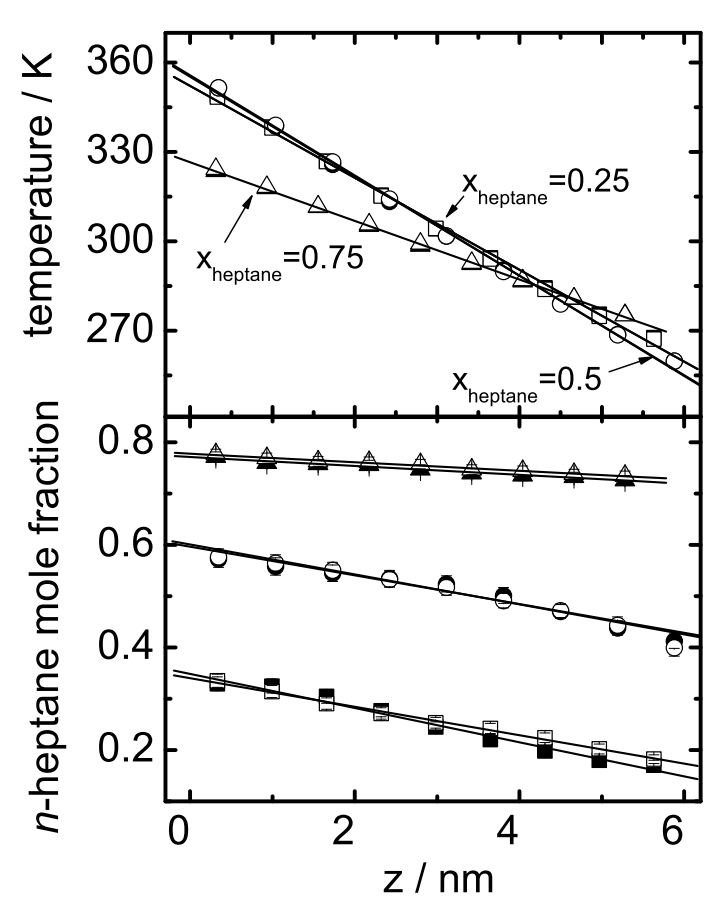

FIG. 3: The temperature and mole fraction profiles for $n$-heptane/benzene mixtures with $x_{n-\text { heptane }}=0.25(\square, \boldsymbol{\square}), 0.5(\circ, \bullet)$ and $0.75(\triangle, \mathbf{\Delta})$. The solid and open symbols refer to 9 slabs of the downward and upward branch in the simulation box. The error bars in the temperature profile are smaller than the symbols, which is also reflected by the overlapping points for the upward and downward branch. The maximum error bar in the concentration profile does not exceed two symbols sizes.

reproduces the experimental trend very well, however the simulated values are systematically $\approx 3 \times 10^{-3} \mathrm{~K}^{-1}$ lower than in the experiment. The same trend was observed by Zhang et $\mathrm{al}^{11}$ for benzene/cyclohexane mixture, they found that the simulated Soret coefficient was $4 \times 10^{-3} \mathrm{~K}^{-1}$ lower than in experiment. We also investigated the influence of the exchange number on the Soret coefficient for the $n$-heptane mole fraction of 0.25 . Increasing the exchange number from 100 to 200 makes the temperature gradient roughly two times smaller. For $N_{\text {exch }}=200, x_{\text {heptane }}=0.25$ and the same simulation time we have found $S_{\mathrm{T}}=-(1.25 \pm$ $0.15) \times 10^{-2} \mathrm{~K}^{-1}$ which is roughly $29 \%$ lower than $S_{\mathrm{T}}=-(9.38 \pm 0.88) \times 10^{-3} \mathrm{~K}^{-1}$ obtained for $N_{\text {exch }}=100$. For the benzene/cyclohexane mixture with a molar fraction $x_{\text {benzene }}=0.25$ $S_{\mathrm{T}}$ for $N_{\text {exch }}=200$ had also been found to be $46 \%$ lower than for $N_{\text {exch }}=100$ (Ref. 11). 


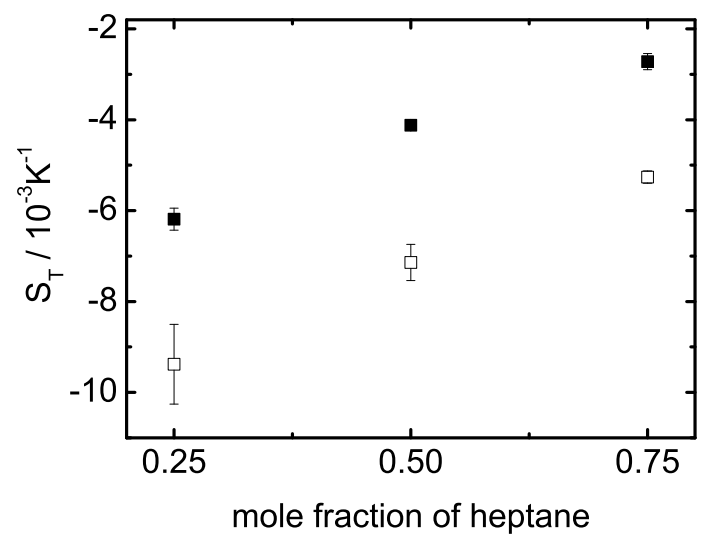

FIG. 4: Comparison of the simulated Soret coefficients $(\square)$ of $n$-heptane in benzene with the experimental values $(\boldsymbol{\square})^{14}$.

We also checked the influence of the size of the rectangular simulation box $\left(L_{\mathrm{x}}=L_{\mathrm{y}}\right.$ $\left.=L_{\mathrm{z}} / 3\right)$ on the Soret coefficient for equimolar $n$-heptane/benzene mixture. The obtained value for 960 molecules in the simulation box and $N_{\text {exch }}=100$ is $(7.14 \pm 0.4) \times 10^{-3} \mathrm{~K}^{-1}$ after $10.5 \mathrm{~ns}$. This result is in perfect agreement with $S_{\mathrm{T}}=(7.16 \pm 0.22) \times 10^{-3} \mathrm{~K}^{-1}$ (c.f. Fig. 4) obtained for 1500 molecules in simulation box and the same exchange number averaged over 13.7ns. Therefore, we can conclude that our simulation system is large enough.

All simulations for branched heptanes (3-MH, 2,3-MH and 2,4-DMP) in benzene were performed for 960 molecules in the simulation box and with an exchange rate of $N_{\text {exch }}=100$. Fig. 5 shows the temperature and mole fraction profiles for mixtures of the three branched heptanes in benzene averaged over 10.5 ns. Fig. 6 shows the calculated Soret coefficient in comparison with experimental data ${ }^{14}$. The RNEMD reproduces experimental trend very well, however the simulated values are systematically $\approx 3 \times 10^{-3} \mathrm{~K}^{-1}(\approx 25 \%)$ lower than in experiment. For 2,3-DMP/benzene system with the smallest Soret coefficient we performed a second, independent MD simulation with a simulation time of $4.5 \mathrm{~ns}$. The magnitude of the obtained Soret coefficient $S_{\mathrm{T}}=3.2 \times 10^{-3} \mathrm{~K}^{-1}$ agrees within the error bars with the value $S_{\mathrm{T}}=(3.04 \pm 0.2) \times 10^{-3} \mathrm{~K}^{-1}$ obtained after $10.5 \mathrm{~ns}$. 


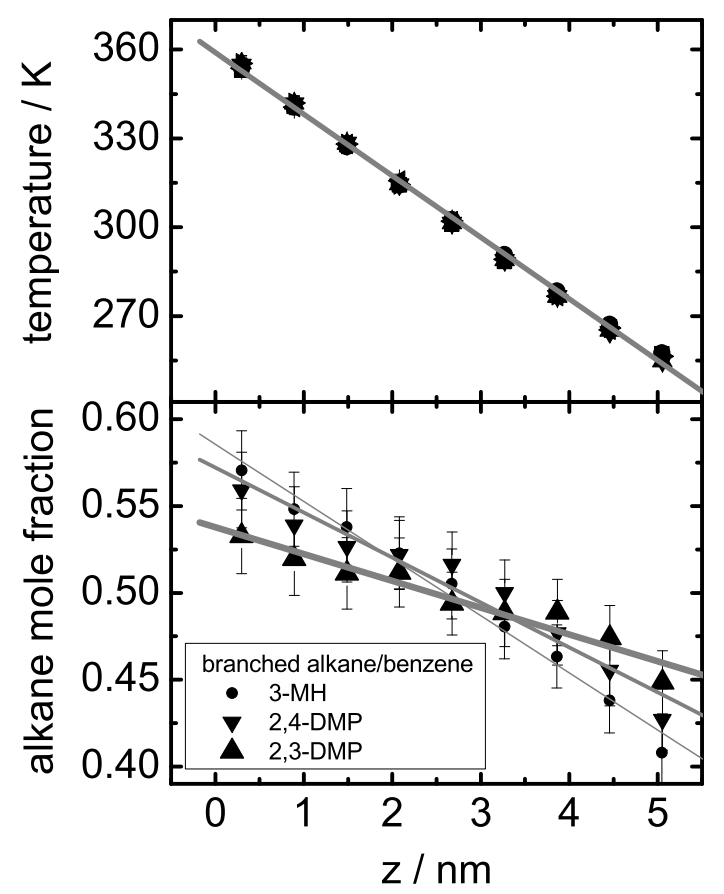

FIG. 5: Temperature and mole fraction profiles for equimolar mixtures of 3-MH, 2,3-DMP and 2,4-DMP in benzene.

\section{DISCUSSION}

In both experiment and simulation we found for equimolar mixtures of heptane isomers and benzene that the magnitude of Soret coefficient decreases with increasing degree of branching. This result can be analyzed in terms of the work of Reith and Müller-Plathe ${ }^{6}$. Generally, there is no Soret effect in the mixture of absolutely equal components due to the principle of symmetry. The Soret effect is basically the response of the system to the difference between two mixing partners. In their work, they considered binary equimolar mixtures of Lennard Jones particles. The influence of the difference in mass, $m$, diameter, $\sigma$, and depth of the interaction potential, $\varepsilon$ on the Soret coefficient was investigated. They varied systematically the ratio of one of the parameters (e.g. $\left.m_{1} / m_{2}\right)$ while keeping the two other parameters fixed and equal. By this procedure the obtained three additive contributions $S_{\mathrm{T}}^{m}, S_{\mathrm{T}}^{\sigma}$ and $S_{\mathrm{T}}^{\varepsilon}$ of the total Soret coefficient $S_{\mathrm{T}}^{L J}$ stemming from the difference in mass, diameter and interaction strength, respectively

$$
S_{\mathrm{T}}^{L J}=S_{\mathrm{T}}^{m}+S_{\mathrm{T}}^{\sigma}+S_{\mathrm{T}}^{\varepsilon} .
$$




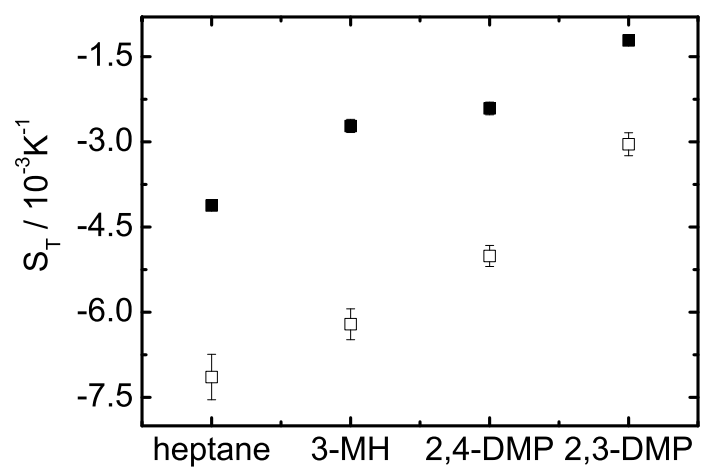

FIG. 6: Comparison of the simulated Soret coefficients $(\square)$ with the experimental values $(\boldsymbol{\square})^{14}$ for equimolar mixtures of $n$-heptane and its isomers in benzene.

TABLE II: The mass, the size and the interaction contributions to the Soret coefficient $\left(10^{-3} \mathrm{~K}^{-1}\right)$ calculated using Eq. 6, 7 and 8. The sum of these three contributions $\mathrm{S}_{T}^{L J}$ is compared with simulation results $\mathrm{S}_{T}^{\text {sim }}$.

\begin{tabular}{|l|r|r|r|r|l|}
\hline mixture & $S_{T}^{m}$ & $S_{T}^{\sigma}$ & $S_{T}^{\varepsilon}$ & $\mathrm{S}_{T}^{L J}$ & $\mathrm{~S}_{T}^{\text {sim }}$ \\
\hline n-heptane/benzene & 2.23 & -5.72 & 0.11 & -3.38 & -7.14 \\
3-MH/benzene & 2.23 & -5.72 & -1.68 & -5.17 & -6.21 \\
2,4-DMP/benzene & 2.23 & -5.72 & -3.04 & -6.53 & -5.01 \\
2,3-DMP/benzene & 2.23 & -5.72 & -3.54 & -7.03 & -3.04 \\
\hline
\end{tabular}

Results indicate that heavier species, smaller species, and species with higher interaction strengths tend to accumulate in the cold region The following empirical laws were obtained by low order fits of the independent parameter variations

$$
\begin{array}{cc}
S_{\mathrm{T}}^{m}=-0.7\left(\frac{m_{1}}{m_{2}}\right)^{2}+9.5\left(\frac{m_{1}}{m_{2}}\right)-8.8 & \text { for } \frac{m_{1}}{m_{2}} \leq 8 \\
S_{\mathrm{T}}^{\sigma}=67.4\left(\frac{\sigma_{1}}{\sigma_{2}}\right)^{2}-179.3\left(\frac{\sigma_{1}}{\sigma_{2}}\right)+111.9 & \text { for } \frac{\sigma_{1}}{\sigma_{2}} \leq 1.25 \\
S_{\mathrm{T}}^{\varepsilon}=4.4\left(\frac{\varepsilon_{1}}{\varepsilon_{2}}\right)^{2}+3.5\left(\frac{\varepsilon_{1}}{\varepsilon_{2}}\right)-7.9 \quad \text { for } \frac{\varepsilon_{1}}{\varepsilon_{2}} \leq 1.75
\end{array}
$$

Table II shows the mass, the size and the interaction contribution to the Soret coefficient. The sum of these three values $S_{T}^{L J}$ is compared with $S_{\mathrm{T}}$ from simulations. The mass ratio of heptanes to benzene $\left(\mathrm{m}_{1} / \mathrm{m}_{2}=1.28\right)$ is obviously not sensitive to the degree of branching, 
so that $S_{\mathrm{T}}^{m}>0$ is the same for all four mixtures. The size contribution $\mathrm{S}_{\mathrm{T}}^{\sigma}$ can also be considered the same as far as the density of the pure branched heptanes agree within $2 \%$ with the density of pure $n$-heptane (c.f. Tab. I). Quantitatively, this contribution can be estimated from the Van-der-Waals volumes $V^{V d W}$ of two mixing partners, considering $\sigma_{1}$ $\sigma_{2}^{-1}=\left(\mathrm{V}_{1}^{V d W} / \mathrm{V}_{2}^{V d W}\right)^{1 / 3}$. The Van-der-Waals volume of a molecule can be determined by atomic increments ${ }^{26}$. The Van-der-Waals volume of benzene $48.4 \mathrm{~cm}^{3} \mathrm{~mol}^{-1}$ is less than the Van-der-Waals volume of $n$-heptane, 3-MH, 2,3-DMP, 2,4-DMP (78.49, 78.48, 78.47, 78.47 $\mathrm{cm}^{3} \mathrm{~mol}^{-1}$ ) (Ref. [25]). It turns out, that the size contribution $S_{\mathrm{T}}^{\sigma}$ is negative and not sensitive to the degree of branching. The third term in Eq. $5 S_{\mathrm{T}}^{\varepsilon}$ can be estimated from the vaporization enthalpies of the pure components, considering $\varepsilon_{1} / \varepsilon_{2}=\mathrm{H}_{1}^{v a p} / \mathrm{H}_{2}^{v a p}$ (Ref. [13]). For calculation of $S_{\mathrm{T}}^{\varepsilon}$ we used the vaporization enthalpies from the simulations (c.f. Tab. I). Thus, the (constant) size contribution $S_{\mathrm{T}}^{\sigma}<0$ dominates the (constant) mass contribution $S_{\mathrm{T}}^{m}>0$ making $S_{\mathrm{T}}$ negative. $\mathrm{S}_{T}^{L J}$ calculated in this way varies only because of the interaction strength differences and it was found to be a decreasing function of degree of branching, what contradicts to the trend, observed by simulation and experimentally. It is therefore clear that a simple analysis which maps the complex molecules onto simple Lennard-Jones particles is not sufficient. This is consistent with earlier results on benzene-alkane mixtures ${ }^{11}$. The different nature of the benzene-benzene and alkane-alkane interactions due to the benzene quadrupole moment could also contribute to the Soret coefficient. However, the quantitative estimation of this contribution is difficult. The branching effect, probably, can be considered by taking into account the non - ideality of alkane/benzene mixtures caused by their anisotropy in shape.

There is another way of interpreting the Soret effect in terms of simple molecular properties: the moments of inertia have been shown to make an important contribution to the Soret effect for mixtures of cyclohexane and benzene isotopes ${ }^{27}$. Debuschewitz and Köhler found that the Soret coefficient could be written as a sum of three contributions:

$$
S_{T}=a_{M} \delta M+b_{I} \delta I+S_{T}^{0}
$$

where $\delta M=\left(M_{1}-M_{2}\right)\left(M_{1}+M_{2}\right)^{-1}$ and $\delta I=\left(I_{1}-I_{2}\right)\left(I_{1}+I_{2}\right)^{-1}$ are the relative differences of the masses $\left(M_{1}, M_{2}\right)$ and moments of inertia $\left(I_{1}, I_{2}\right)$ of the molecules, respectively. A further investigation of the isotope effect ${ }^{28}$ suggested that the absolute rather than the relative differences between the masses and moments of inertia should enter the expression 
for the Soret coefficient so that the difference terms in Eq. (9) are given by $\delta M=M_{1}-M_{2}$ and $\delta I=I_{1}-I_{2}$. In our previous work ${ }^{14}$ we have calculated the moments of inertia about the symmetry axis ( $I_{z z}$ for the disk-like benzene molecules, $I_{x x}$ for the $n$-heptane and five heptane isomers, including 3-MH, 2,3-DMP and 2,4-DMP) using an atomistic model for single molecules in vacuum ${ }^{29}$. It was found that the Soret coefficient correlates almost increasing linearly with the moment of inertia $I_{x x}$ in the direction of the main chain. Based on this observation one might conclude that the Soret coefficient is completely determined by the moment of inertia, but simultaneously with the change of the moment of inertia (kinetic contribution) also the anisometry (static contribution) of the molecule changes. The chemical contribution $S_{T}^{0}$ (c.f. Eq. 9) can be associated with the size (Eq. 7) and the interactions (Eq. 8) effects. Reith and Müller-Plathe ${ }^{6}$ have shown that it is possible to combine Eq. 7 and 8 into a single one which involves the cohesive energy densities $e=\varepsilon / \sigma^{3}$. In our case this parameter is not sensitive to the mixture (c.f. Tab 2), so that the chemical contribution $S_{T}^{0}$ (c.f. Eq. 9) turns out to be the same for all four equimolar heptane/benzene mixtures, if we consider experimental evaporization enthalpies $H_{\text {exp }}^{v a p}$ (c.f. Table I).

The shape of the molecules influences their packing. The packing can be analyzed using the radial distribution functions (RDF). Fig. 7A shows the centre-of-mass RDF for pure n-heptane, 3-MH, 2,3-DMP, 2,4-DMP and benzene. The characteristic distance $r$ at which fluctuations in RDF disappear as well as the amplitude and the position of the first maximum become larger with increasing branching and correlate with the magnitude of the Soret coefficient (c.f. Fig. 6). For pure benzene the packing is even closer to the spherical packing than for alkanes. The RDF, calculated between centers of mass of benzene and alkanes in the equimolar alkane/benzene mixtures (c.f. Fig. 7B) shows the same trend as the center of mass RDF, calculated for pure components (c.f. Fig. 7A). The packing efficiency can also be analyzed in terms of the smallest distance between centers of masses of molecules, which can be estimated from the integral of RDF (c.f. Fig. 8). The $n$-heptane- $n$-heptane distance in the pure state and the $n$-heptane-benzene distance in the equimolar mixture are almost equal to each other and less than the benzene-benzene distance in pure state. This means that the benzene rings orient parallel to the $n$-heptane chains and do not disturb the packing of $n$-heptane significantly. In the pure state benzene molecules arrange themselves in a Tshape geometry ${ }^{16}$ resulting in a larger distance between the centers of masses. The behavior of the branched heptanes is different due to their increasingly globular shape. The alkane- 


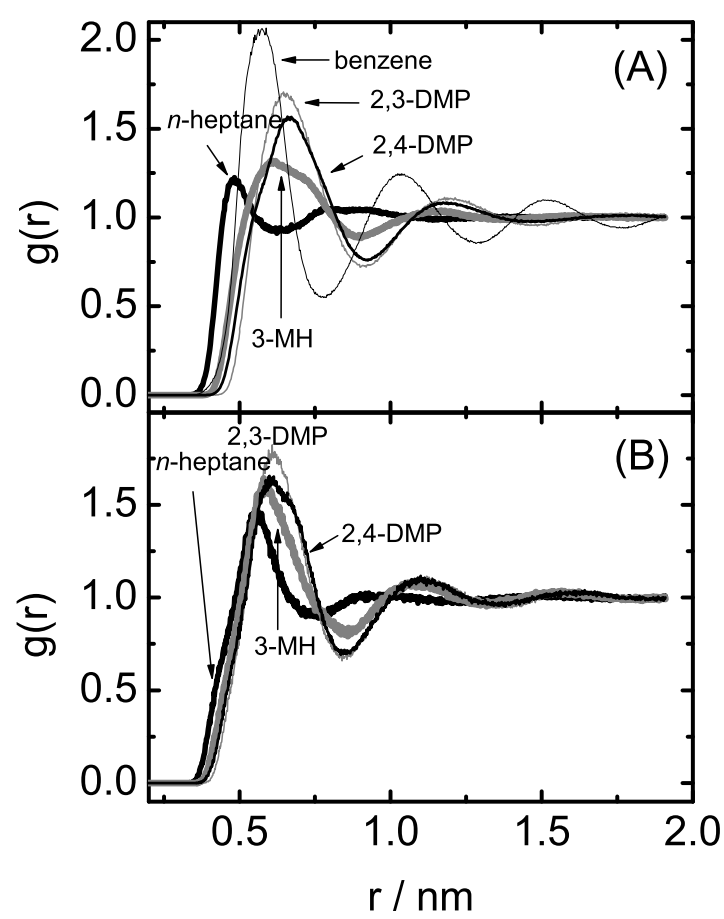

FIG. 7: (A) Center of mass radial distribution function for pure components. (B) Center of mass alkane-benzene radial distribution function for equimolar mixtures.

alkane distance in pure liquid and benzene-alkane distance in the mixture becomes larger with increasing branching and correlates also with the magnitude of the Soret coefficient (c.f. Fig. 6).

The packing effect can influence the diffusion properties of the mixtures. For heptane/benzene mixture the tracer diffusion coefficient of benzene is smaller than the tracer diffusion coefficient of heptane for all investigated concentrations (c.f. Fig. 1 B). At the same time their differences decrease with increasing concentration of heptane. This could be due to the higher packing of benzene with heptane molecules in the heptane rich region.

\section{CONCLUSION}

We applied a equilibrium molecular dynamics and the reverse nonequilibrium moleculardynamic algorithm to calculate the mutual diffusion, tracer diffusion and Soret coefficients in different alkane/benzene mixtures. In order to explore the concentration influence we 


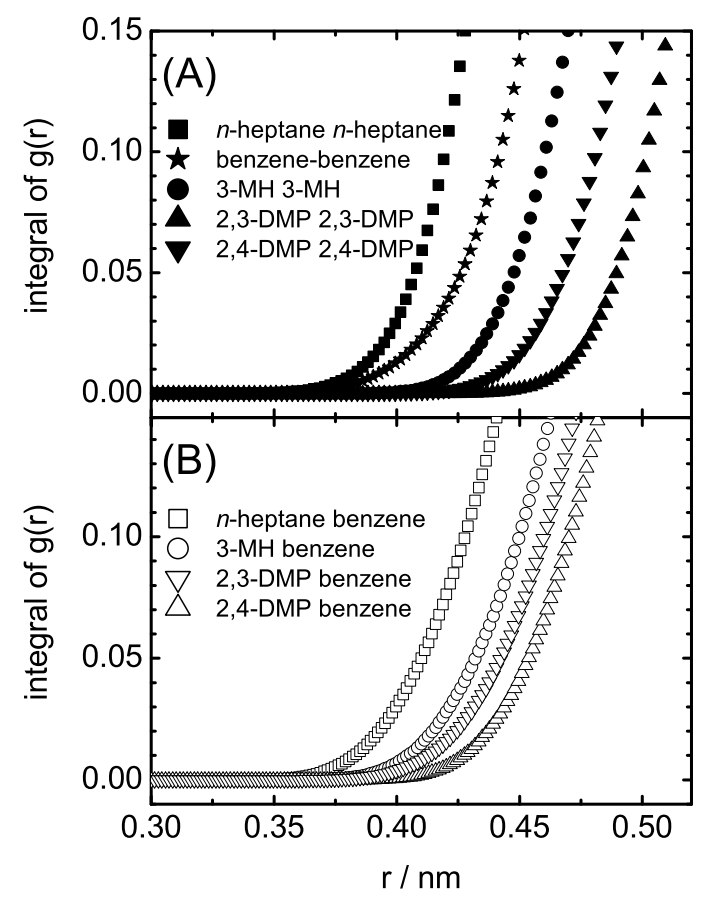

FIG. 8: Integral of the center of mass radial distribution function for pure components and for equimolar mixture between benzene and alkanes.

studied $n$-heptane/benzene mixture at different concentrations. Additionally, we looked into the influence of the degree of branching by investigating equimolar mixtures of the branched heptanes (3-MH, 2,3-DMP, 2,4-DMP ) in benzene. The simulated Soret and mutual diffusion coefficients show the same trend as in experiment. However, the simulated values of $S_{\mathrm{T}}$ values are systematically $\approx 3 \times 10^{-3} \mathrm{~K}^{-1}$ lower than in the experiment. The observed decreasing of the magnitude of $S_{\mathrm{T}}$ for equimolar alkane/benzene mixtures with branching of the alkane can not be explained by mass and size effects. Nevertheless we observe a linear increase of $S_{\mathrm{T}}$ with increasing moment of inertia, which could be purely to kinetic but also due to static contributions due to simultanous change of the anisometry of the molecules. The effect of the molecular shape, which affects the liquid structure, as well as kinetic properties of the mixture, needs to be considered additionally. We have, however, not found a simple relation to take branching or, more generally, molecular shape, into account. 


\section{ACKNOWLEDGMENTS}

We would like to thank Mohammad Alaghemandi, Elena Algaer, Eddie Rossinsky and Thomas Müller for technical help and helpful discussion. We thank the Deutsche Forschungs-

gemeinschaft (WI 1684/3-3) and the John von Neumann Institute for Computing at the Forschungszentrum Jülich, which provided the computer time for this study. 
* p.polyakov@fz-juelich.de

$\dagger$ s.wiegand@fz-juelich.de

1 M. E. Schimpf and J. C. Giddings, Macromolecules 20, 1561 (1987).

2 K. Clusius and G. Dickel, Naturwissenschaften 27, 148 (1939).

3 H. C. Helgeson, Pure \& Appl. Chem. 57, 31 (1885).

4 P. Costeseque, D. Fargue, and P. Jamet, in Thermal nonequilibrium phenomena in fluid mixtures, edited by W. Köhler and S. Wiegand (Springer, Berlin, 2000), Lecture Notes in Physics, pp. 389-427.

5 D. E. Rosner, R. S. Israel, and B. La Mantia, Combustion and Flame 123, 547 (2000).

6 D. Reith and F. Müller-Plathe, J. Chem. Phys. 112, 2436 (2000).

7 G. Galliero, B. Duguay, J. P. Caltagirone, and F. Montel, Fluid Phase Equilibria 208, 171 (2003).

8 J. M. Simon, D. K. Dysthe, A. H. Fuchs, and B. Rousseau, Fluid Phase Equilibria 151, 151 (1998).

9 G. Galliero, B. Duguay, J. P. Caltagirone, and F. Montel, Philos. Mag. 83, 2097 (2003).

10 A. Perronace, C. Leppla, F. Leroy, B. Rousseau, and S. Wiegand, J. Chem. Phys. 116, 3718 (2002).

11 M. Zhang and F. Müller-Plathe, J. Chem. Phys. 123, 124502 (2005).

12 C. Nieto-Draghi, J. B. Avalos, and B. Rousseau, J. Chem. Phys. 122, 114503 (2005).

13 P. Polyakov, M. Zhang, F. Müller-Plathe, and S. Wiegand, J. Chem. Phys. 127, 014502 (2007).

14 P. Polyakov, J. Luettmer-Strathmann, and S. Wiegand, J. Phys. Chem. B 110, 26215 (2006).

15 J. K. Platten, M. M. Bou-Ali, P. Costeseque, J. F. Dutrieux, W. Köhler, C. Leppla, S. Wiegand, and G. Wittko, Philos. Mag. 83, 1965 (2003).

16 G. Milano and F. Müller-Plathe, J. Phys. Chem. B 108, 7415 (2004).

17 G. Martin and J. Siepmann, J. Phys. Chem. B 102, 2569 (1998).

18 S. Nath and R. Khare, J. Chem. Phys. 115, 10837 (2001).

19 J. Chang and S. Sandler, J. Chem. Phys. 121, 7474 (2004).

20 F. Müller-Plathe, Comput. Phys. Commun. 78, 77 (1993).

21 H. J. C. Berendsen, J. P. M. Postma, W. F. van Gunsteren, A. Di Nola, and J. R. Haak, J. 
Chem. Phys. 81, 3684 (1984).

22 G. Kouris and C. Panayiotou, J. Chem. Eng. Data. 34, 200 (1989).

23 H. Ertl and F. Dullein, J. AIChE 19, 1215 (1973).

24 The molar mass, the density and the boiling point information were taken datasheet for the chemical provided by the company Aldrich. See http://www.sigmaaldrich.com.

25 C. Yaws, Chemical Properties Handbook (McGraw Hill, New York, 1999).

26 J. Edward, J. Chem. Educ. 47, 261 (1970).

27 C. Debuschewitz and W. Köhler, Phys. Rev. Lett, 87, 055901 (2001).

28 G. Wittko and W. Köhler, J. Chem. Phys. 123, 014506 (2005).

29 Chem3d, ver. 10 (2006). 